\title{
Untersuchungen aus dem chemischen Laboratorium von Prof. Alexander Saytzeff zu Kasan.
}

\section{Beitrag zur Kenntniss der Jalapinolsäure; von}

N. Kromer.

Das Harz der sog. falschen Jalape wird, wie frühere Untersuchungen ${ }^{1}$ ) dargethan haben, bei der Hydrolyse mit Mineralsäuren unter anderen Produkten in eine fettartige Säure, welche Jalapinolsäure benannt worden ist, zerlegt.

Sowohl die Zusammensetzung der genannten Säure als auch die Zersetzungen und Umwandlungen, welche sie durch einige Agentien erfährt, sind nicht genügend erforscht; auch konnten bezüglich ihrer Reinheit, wie sie zur Analyse verwandt worden war, Zweifel laut werden.

Demgemäss lasse ich gegenwärtig die Untersuchung über einige Derivate der Jalapinolsäure, welche geeignet sind, einiges Licht über die Constitution derselben zu verbreiten, folgen. Zur Darstellung der Säure diente ein falsches Jalapenharz, das ich der Liebenswurdigkeit der Firma Zimmer \& Co. in Frankfurt a./M. verdanke.

Dasselbe wurde zuerst mit Barythydrat behandelt und im Einzelnen so verfahren, wie es bereits früher ${ }^{2}$ ) von mir mitgetheilt wurde.

Die bei dieser Behandlung erhaltene Jalapinsäure, welche zur Reinigung mehrmals aus alkoholischer Lösung durch Aether gefällt worden war, wurde mit verdünnter Salzsäure gekocht, und die neben Glycose gewonnene, in Wasser unlösliche Jalapinolsäure durch Waschen mit Wasser von anhaftender Mineralsäure befreit.

Die erhaltene Jalapinolsäure war gefärbt und konnte von färbenden Bestandtheilen dadurch befreit werden, dass eine alkoholische Lösung derselben mit einer zur Fällung der Säure unvollständigen Quantität einer gesättigten wässrigen Barythydratlösung fractionirt gefällt wurde.

1) Zeitschr. des allgem. österr. Apothekervereins Nr. 18/24. 1895.

2) A. a. 0 . 
Kromer: Beitrag z. Kenntniss der Jalapinolsäure. 449

Diese Reinigungsmethode, sowie diejenige, welche auf der fractionirten Fällung der Fettsäuren mit Magnesiumacetat beruht, konnten nicht befriedigen, da sie nicht unbedeutende Verluste von Material zur Folge hatten und sich nicht geeignet erwiesen, die Einheitlichkeit der Substanz klarzulegen.

Die geeigneteste Methode, die Jalapinolsäure rein zu gewinnen, war, sie in ibre Methyl- und Aethylester, welche gut krystallisirten, überzuführen und aus diesen dann durch Verseifung die Säure zu gewinnen.

Zur Darstellung der Ester wurden je 100 Grm. der Säure in der zweifachen Menge der entsprechenden Alkohole gelöst und unter Eiskühlung so lange trockner Chlorwasserstoff hineingeleitet, bis derselbe nicht mehr absorbirt wurde.

Nach 48stündigem Stehen wurde die Mischung in Eiswasser gegossen und der sich abscheidende Ester mit Wasser gewaschen. Nach mehrmaligem Umschmelzen über Wasser und Trocknen zwischen Fliesspapier wurden die Ester zuerst aus neutralem niedrig siedendem Petroläther, alsdann aus Aether umkrystallisirt.

Das Umkrystallisiren wurde so lange fortgesetzt, bis Aether und Petroläther bei der Krystallisation keine Schwankungen in den Schmelzpunkten der betreffenden Ester hervorriefen.

Der Methylester krystallisirt in glänzenden, farblosen Blättchen, während der Aethylester in derben, nadelförmigen, einige Centimeter langen Gebilden erhalten wurde.

Die Bestimmung der Schmelzpunkte, welche im Capillarrohre ausgeführt wurde, lieferte folgendes Resultat:

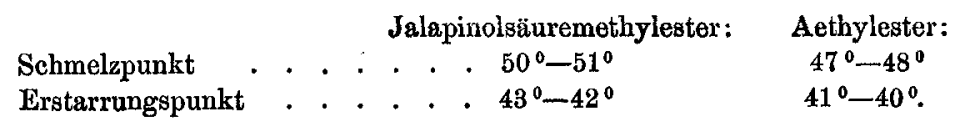

Die Verbrennung der vorher geschmolzenen, alsdann im Exsiccator über Schwefelsäure getrockneten Substanzen mit Kupferoxyd führte zu folgenden Werthen:

0,1988 Grm. des Methylesters lieferten 0,2165 Grm. $\mathrm{H}_{2} \mathrm{O}$ u. 0,521 Grm. $\mathrm{CO}_{2}=12,09 \% \mathrm{H}$ und $71,46 \% \mathrm{C}$.

$0,1707 \mathrm{Grm}$. desselben gaben 0,185 Grm. $\mathrm{H}_{2} \mathrm{O}$ u. 0,4451 Grm. $\mathrm{CO}_{2}$ $=12,04 \%$ H. u. $71,10 \%$.

$0,1842 \mathrm{Grm}$. des Aethylesters gaben 0,204 Grm. $\mathrm{H}_{2} \mathrm{O}$ u. 0,4849 Grm. $\mathrm{CO}_{\mathbf{2}}=12,3 \% \mathrm{HH}$ u. $70,78 \%$ C.

Journal f. prakt. Chemie [2] Bd. 57. 
450 Kromer: Beitrag z. Kenntniss der Jalapinolsäure.

Berechnet für

Gefunden:

$\mathrm{C}_{15} \mathrm{H}_{30}(\mathrm{OH}) \mathrm{CO}_{2} \cdot \mathrm{CH}_{3}$ : $\mathrm{C}_{15} \mathrm{H}_{80}(\mathrm{OH}) \mathrm{CO}_{2} \cdot \mathrm{C}_{2} \mathrm{H}_{5}$ : Methylester: Aethylester:

$\begin{array}{llllll}\text { C } & 71,32 & 72 & 71,46 & \mathbf{7 1 , 1 0} & \mathbf{7 1 , 7 8} \%\end{array}$

H $11,88 \quad 12 \quad 12,09 \quad 12,04 \quad 12,30 \ldots$.

Zur Verseifung der Ester wurden dieselben mit titrirter, alkoholischer Kalilauge 1 Stunde lang auf dem Dampfbade am Rückflussrohre gekocht und der Ueberschuss des angewandten Alkalihydrats zurückgemessen.

20 Cem. der alkoholischen Lauge wurden durch $27,9 \mathrm{Ccm}, \mathrm{n} / 10^{\circ}$ Oxalsäure sowie durch $26,8 \mathrm{Ccm}$, einer Salzsäure gesättigt.

$0,4135 \mathrm{Grm}$. des Aethylesters wurden mit $20 \mathrm{Ccm}$. obiger Kalilauge gekocht und erforderten bei der Rücktitration 12,8 Ccm. Salzsäure; mithin wurden $0,079298 \mathrm{Grm}$. $\mathrm{KHO}$ oder $19,17 \% \mathrm{KHO}=13,35 \% \mathrm{~K}$ verbraucht.

2,064 Grm. des Aethylesters wurden mit $80 \mathrm{Ccm}$. alkoholischer Kalilauge gekocht, von der $1 \mathrm{Ccm}$. 0,0077556 Grm. KHO enthielt und mit Salzsäure zurücktitrirt, von der $1 \mathrm{Ccm}$. 0,0058754 Grm. KHO sättigte; verbraucht wurden zur Rücktitration $39,2 \mathrm{Ccm}$. $\mathrm{HCl}$; somit waren 0,3901323 Grm. KHO $=18,90 \%$ KHO oder $13,16 \%$ K erforderlich gewesen.

Berechnet fürr

Gefunden:

$\mathrm{C}_{15} \mathrm{H}_{30}(\mathrm{OH}) \mathrm{CO}_{2} \cdot \mathrm{CH}_{8}: \mathrm{C}_{15} \mathrm{H}_{30}(\mathrm{OH}) \mathrm{CO}_{2} \cdot \mathrm{C}_{2} \mathrm{H}_{5}$ : Methylester: Aethylester : KHO $19,30 \quad 18,73 \quad 19,17 \quad 18,73 \%$.

Die bei der Verseifung abgeschiedene Säure schmolz bei $67^{\circ}-68^{\circ}$ und war optisch inactiv; das durch Neutralisation der Jalapinolsäure mit Ammoniak und darauffolgende Fällung mit Silbersalpeter erhaltene Silbersalz der Säure, welches ziemlich lichtbeständig war, gab nach dem Trocknen im Exsiccator bei der Metallbestimmung folgende Werthe:

0,3171 Grm. der Silberverbindung hinterliessen 0,0896 Grm. Ag = $28,25 \%$ Ag.

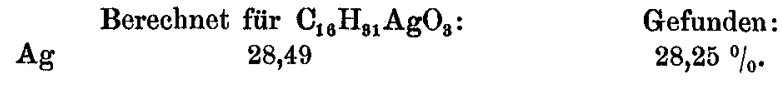

Einwirkung von Essigsäureanhydrid auf Jalapinolsäure.

Zum Nachweise von vorhandenen Hydroxylgruppen wurde die freie Säure mit geschmolzenem Natriumacetat und Essigsäureanhydrid gekocht, wobei ein schmieriges Produkt erhalten wurde, das schwer rein erhalten werden konnte.

Vollständig gelang der Ersatz der Hydroxylgruppe durch 
Kromer: Beitrag z. Kenntniss der Jalapinolsäure. 451

einen Säurerest, wenn an Stelle der freien Jalapinolsäure ihr Methyl- oder Aethylester gewählt wurde.

$\mathrm{Zu}$ diesem $\mathrm{Z}$ wecke wurde der Aethylester mit geschmolzenem Natriumacetat und überschüssigem Essigsäureanhydrid im Einschlussrobre einige Stunden lang auf ca. $180^{\circ}$ erhitzt und daranf der Röhreninhalt in einer Porzellanschaale auf dem Wasserbade von unverändertem Essigsäureanhydrid und etwaig vorhandener freier Essigsäure durch Abdampfen befreit.

Die Acetylverbindung wurde hierauf mit Petroläther aufgenommen und hinterblieb nach dem Verdunsten desselben als eine hellgelb gefärbte, ölige Masse, die neutral reagirte und in Aether, Petroläther, Alkohol leicht, in Wasser aber unlöslich war. Durch Kalihydrat wurde sie verseift und schied nach dem Ansäuern der alkalischen Lösung die freie Jalapinolsãure $a b$.

Bei $224^{\circ}-225^{\circ}$ und $50 \mathrm{Mm}$. Druck destillirte die Aethylverbindung unverändert.

Zur Bestimmung der in der Verbindung enthaltenen Acetylgruppe wurde die Methode der directen Titration und die der Destillation angewandt.

1,306 Grm. der Acetylverbindung, welche im Exsiccator getrocknet worden war, wurden mit $60 \mathrm{Ccm}$. alkoholischer Kalilauge, von der $1 \mathrm{Ccm}$. 0,00899 Grm. KHO enthielt, anf dem Wasserbade 4 Stunden lang gekocht und mit Salzsäure, von der $1 \mathrm{Ccm}$. 0,00591447 Grm. KHO sättigte, zurücktitrirt; hierzu waren $18,2 \mathrm{Ccm}$. Salzsäure erforderlich; mithin waren $0,43175 \mathrm{Grm}$. KHO $=33,82 \%$ verbracht worden.

Zur directen Bestimmung der Acetylgruppe wurden 2,55 Grm. der: Verbindung mit Kalibydrat verseift und der Rückstand nach dem Uebersättigen mit Phosphorsäure der Destillation mit Wasserdämpfen so lange unterworfen, bis das Destillat neutral reagirte.

Das gewonnene Destillat erforderte zur Sättigung 7,4 Ccm. " $7 / 1-\mathrm{KHO}$ $=0,444$ Grm. $\mathrm{C}_{2} \mathrm{H}_{4} \mathrm{O}_{2}$ oder $17,41 \%$.

Wird von dem im ersten Falle gewonnenen Werthe die Menge $\mathrm{KHO}$ in Abzug gebracht, welche dem Alkoholrest entspricht, so resultiren für das Acetyl 14,92\% $\mathrm{KHO}$.

1. $\mathrm{KHO}$

Berechnet für $\mathrm{C}_{15} \mathrm{H}_{30} \mathrm{O}\left(\mathrm{CH}_{3} \mathrm{CO}\right) \mathrm{CO}_{2} \cdot \mathrm{C}_{2} \mathrm{H}_{8}$ :

2. $\mathrm{C}_{2} \mathrm{H}_{4} \mathrm{O}_{2}$

$$
32,86
$$

17,54
Gefunden:

$33,82 \%$

17,41, .

Einwirkung ron Brom auf Jalapinolsäure.

Da die Analysen der erhaltenen Ester nicht im Stande sind, die Frage zu entscheiden, ob der Jalapinolsäure, wie es 
angenommen worden war, die Formel $\mathrm{C}_{16} \mathrm{H}_{30} \mathrm{O}_{3}$, welche einer ungesättigten Verbindung entspricht, zukommt oder ob ihr die Zusammensetzung, welche einer dieser Formel entsprechenden gesättigten Säure zukommt, beigelegt werden muss, wurde eine Behandlung der Säure mit Brom ausgeführt.

Hierbei erwies es sich, dass, wenn Jalapinolsäure, welche vorher in Eisessig oder Chloroform gelöst ist, mit einer Bromlösung in den angegebenen Lösungsmitteln versetzt wird, keine Entfärbung des Broms stattfindet, dass aber die Jalapinolsäure bei längerer Einwirkung von Brom eine beträchtliche Menge des Halogens aufzunehmen vermag.

Wurde Jalapinolsäure in Eisessig gelöst, mit so viel Brom versetzt, dass die Mischung nach 48 stündigem Stehen noch braun gefärbt war, und alsdann mit Wasser verdünnt, so schieden sich ölige Tropfen einer Säure aus, die sowohl von Aether, als auch von Petroläther leicht aufgenommen wurden.

Dass hierbei eine Bromaufnahme stattgefunden hatte, wurde einerseits durch die Beilstein'sche Reaction, andererseits durch die Natriumschmelze und nachfolgendes Ansäuern mit Salpetersäure und Fällen mit Silbernitrat dargethan.

Es blieb somit die Frage offen, ob hier eine Addition oder Substitution durch Halogen vorliegt.

Um die Quantität von Brom zu ermitteln, welche die Jalapinolsäure aufnimmt, und den Einfluss des Lösungsmittels, welchen dieses auf die Bromaufnahme ausübt, darzuthun, wurde die Säure sowohl in einer Lösung von Chloroform, als auch in Eisessig mit einem Ueberschuss von Brom versetzt und bei gewöhnlicher Temperatur 48 Stunden lang stehen gelassen.

Die Quantität von Brom wurde so gewählt, dass auch nach der angegebenen Zeitdauer die Mischung noch freies Brom enthielt.

Aus dieser wurde das bromhaltige Produkt durch Wasser abgeschieden, und nachdem dasselbe durch Aether, Petroläther oder Chloroform extrahirt worden war, wurde die betreffende Ausschüttelungsflüssigkeit so lange mit kaltem Wasser gewaschen, bis dieses keine saure Reaction mehr erkennen liess.

In allen Fällen wurde das Auftreten von grossen Mengen von Bromwasserstoff wahrgenommen. 
Kromer: Beitrag z. Kenntniss der Jalapinolsäure. 453

0,5014 Grm. des Bromproduktes, welches in Chloroformlösung erhalten wurde, gaben nach Carius $0,3888 \mathrm{Grm}$. $\mathrm{AgBr}=32,99 \% \mathrm{Br}$.

$0,5820 \mathrm{Grm}$. eines Bromproduktes, das aus Eisessiglösung abgeschieden worden war, gaben $0,4256 \mathrm{Grm}$. $\mathrm{AgBr}=31,11 \% \mathrm{Br}$.

$0,6948 \mathrm{Grm}$. eines Bromproduktes, das bei 96 stündiger Einwirkung von Brom auf Jalapinolsäure, die in Eisessig gelöst war, erhalten wurde, gaben 0,58 Grm. $\mathrm{AgBr}=35,52 \% \mathrm{Br}$.

Aus den vorstehenden Zahlen ist ersichtlich, dass sich die Bromaufnahme der Jalapinolsäure, was die Dauer der Einwirkung und den Einfluss des Lösungsmittels anbetrifft, in engen Grenzen bewegt, dass aber die Quantität des aufgenommenen Halogens keineswegs constant genannt werden kann.

Alle Bromprodukte waren hellgelb gefärbt, bei gewöhnlicher Temperatur flüssig, erstarrten aber beim Abkühlen zu porzellanartigen Massen. Beim Aufbewahren im Vacuumexsiccator über Aetzkali und Schwefelsäure zersetzten sie sich unter Abgabe von Bromwasserstoff.

Versuche, das halogenhaltige Produkt durch Destillation zu reinigen, schlugen fehl, ebenso führte auch das Bestreben, durch Darstellung von Kalisalzen zum Ziele zu gelangen, zu keinem Resultat.

Wurde das Bromprodukt mit Kaliumcarbonat versetzt, eine kleine Menge Wasser hinzugegeben, bis Lösung unter Kohlensäureentwicklung eintrat und alsdann mit Alkohol das überschïssig angewandte Kaliumcarbonat ausgefällt, so schied sich beim Verdunsten der alkoholischen Lösung eine recht beträchtliche Menge Bromkalium ab, während die zurückbleibende zähflüssige Substanz sehr wechselnde Quantitäten von Halogen enthielt.

So gaben 0,4042 Grm. der Substanz, welche aus dem obengenannten Produkte mit einem Gehalte von $35,52 \% \mathrm{Br}$ erhalten wurden, nach vorheriger Behandlung mit Kaliumearbonat, bei der Brombestimmung nach Carius 0,1516 Grm. $\mathrm{AgBr}=15,95 \% \mathrm{Br}$.

Wäre die Einwirkung des Broms auf Jalapinolsäure in der Weise vor sich gegangen, dass im ersten Falle 2 Atome Brom eingetreten sind und bei der Behandlung mit Kaliumcarbonat eines dieser Atome abgespalten worden ist, so müssten bei der Behandlung der Fettsäure mit Brom 37,20\% Br (berechnet für $\mathrm{C}_{16} \mathrm{H}_{30} \mathrm{Br}_{2} \mathrm{O}_{3}$ ) oder 22,79\% $\mathrm{Br}$ (berechnet für $\mathrm{C}_{16} \mathrm{H}_{31} \mathrm{BrO}_{3}$ ) erhalten werden. 
454 Kromer: Beitrag z. Kenntniss der Jalapinolsäure.

Um die Quantität des Halogens zu bestimmen, welche die Jalapinolsäure direct aufzunehmen vermag, wurde eine gewogene Menge vorher getrockneter Säure mit einer gewogenen Quantität (entsprechend 2 Atomen) getrockneten Bromes in Chloroformlösung zusammengebracht und 48 Stunden lang der Einwirkung überlassen.

5,05 Grrn. Jalapinolsäure, mit 3,2 Grm. Brom in Reaction gebracht, gaben nach dem Abdestilliren des Chloroforms im Vacuum 7,12 Grm. Gesammtrückstand.

Das abdestillirte Chloroform enthielt ausser freiem Brom bedeutende Mengen Bromwasserstoff. Der Rückstand erstarrte in den Krystallisationen der unveränderten Jalapinolsäure, die von flüssigen Antheilen der bromhaltigen Substanz durchsetzt waren.

Unter den angegebenen Bedingungen hatte die Jalapinolsäure $40,99 \% \mathrm{Br}$ aufgenommen, während, wenn ihr die $\mathrm{Zu}$ sammensetzung $\mathrm{C}_{10} \mathrm{H}_{30} \mathrm{O}_{3}$, die einer ungesättigten Säure entspricht, zukäme, sie $59,25 \% \mathrm{Br}$ hätte direct addiren müssen.

Dass hier die Bromaufnahme nicht unter Addition, sondern unter Ersatz von Wasserstoffatomen stattgefunden hat, beweist das gleichzeitige Auftreten von Halogenwasserstoff.

Hoehnel ${ }^{1}$ ) beschreibt bei Gelegenheit der Untersuchung der Convolvulinolsäure einen Versuch, der in der Einwirkung von Bromwasserstoff auf Jalapinolsäure besteht, und ihm eine gut krystallisirte Bromverbindung vom Schmelzpunkte ca. 10 lieferte.

Eine Brombestimmung ist von ihm nicht ausgeführt worden.

Reduction der Jalapinolsäure mittelst Jodwasserstoff.

Zur Ueberführung der Jalapinolsäure in die sauerstoffärmere Fettsäure leistete die Methode von Alex. Saytzeff, welche auf der directen Einwirkung der zu reducirenden Fettsäure auf Jodphosphor bei Gegenwart von Jodwassersoffsäure beruht, vorzügliche Dienste.

Zu diesem Zwecke wurden $20 \mathrm{Grm}$. des Methylesters auf 40 Grm. Jodphosphor geschüttet, die Mischung mit einer kleinen Menge Jodwasserstoffsäure, an Stelle dieser kann auch

1) Arch. d. Pharm. 234, Heft 9. (1896.) 
Kromer: Beitrag z. Kenntniss der Jalapinolsäure. 455

Wasser angewandt werden, befeuchtet und 24 Stunden lang der Ruhe überlassen; hierauf wurde die Mischung auf dem Wasserbade bis zum völligen Aufhören der Jodwasserstoffentwicklung erwärmt.

Die neben phosphoriger Säure hinterbliebene Jodfettsäure blieb beim Abkühlen der Mischung flüssig, wurde mit Aether aufgenommen und nach dem Verdunsten des Lösungsmittels in alkoholischer Lösung bei Siedehitze mit Zink und Salzsäure reducirt.

Die aus der Alkohollösung gewonnene Fettsäure wurde zur Zerstörung derartiger Verbindungen mit Kalilauge erhitzt, die trockene, pulverförmige Masse mit Aether behandelt und die Säure durch Salzsäure in Freiheit gesetzt.

Nachdem die Säurs einige Mal durch Umschmelzen über Wasser von Mineralsäure befreit worden war, wurde sie aus Petroläther, Aether und Alkohol umkrystallisirt.

Letzteres Lösungsmittel diente dazu, die Einheitlichkeit der Säure darzuthun, was in der Weise geschah, dass dieselbe fractionirt, aus Alkohol krystallisirt und der Schmelzpunkt der crhaltenen Fractionen bestimmt wurde.

Es wurden 6 Fractionen erhalten, die in farblosen, wohlausgebildeten rhombischen Blättchen krystallisirten, bei $65^{\circ}-$ $66^{\circ}$ schmolzen und bei $63^{\circ}-62^{\circ}$ erstarrten.

Eine alkalische Chamäleonlösung wurde durch die Säure nicht entfärbt.

Bei der Verbrennung mit Kupferoxyd wurden folgende Werthe erhalten:

$0,1842 \mathrm{Grm}$. der Säure gaben $0,2117 \mathrm{Grm} . \mathrm{H}_{2} \mathrm{O}$ u. 0,504 Grm. $\mathrm{CO}_{2}$ $=12,76 \%$ H u. $74,61 \%$ C.

0,151 Grm. derselben gaben 0,172 Grm. $\mathrm{H}_{2} \mathrm{O}$ u. 0,41443 Grm. $\mathrm{CO}_{2}$ $=12,65 \% \mathrm{H} \mathrm{u}, 74,82 \% \mathrm{~L}$.

\begin{tabular}{cccc} 
& Berechnet für & \multicolumn{2}{c}{ Gefunden: } \\
& $\mathrm{C}_{16} \mathrm{H}_{32} \mathrm{O}_{2}:$ & 1. & 2. \\
$\mathrm{C}$ & 75,0 & 74,61 & $\mathbf{7 4 , 8 2} \%$ \\
$\mathrm{H}$ & 12,5 & 12,76 & $\mathbf{1 2 , 6 5} \%$
\end{tabular}

Die Silberverbindung der Säure wurde aus dem Natronsalze, welches aus kochendem Alkohol umkrystallisirt worden war, durch Doppelzersetzung mit Silbernitrat als weisser, in Wasser unlöslicher, lichtbeständiger Niederschlag erhalten. 
456 Kromer: Beitrag z. Kenntniss der Jalapinolsäure.

Im Exsiccator über Schwefelsäure getrocknet, gab die Verbindung bei der Metallbestimmung folgendes Resultat:

$0,344 \mathrm{Grm}$. derselben gaben $0,101 \mathrm{Grm} . \mathrm{Ag}=29,36 \% \mathrm{Ag}$.

$0,502 \mathrm{Grm}$, des Salzes lieferten 0,149 Grm. Ag $=29,68 \% \mathrm{Ag}$. Berechnet für

Ag $\quad \mathrm{C}_{16} \mathrm{H}_{31} \mathrm{~A}$

Gefunden:

1. 2.

$29,36 \quad 29,68 \%$.

Die Kohlenstoffbestimmung sowie die Analyse des Silbersalzes ergaben Werthe, welche einer Säure von der empirischen Zusammensetzung $\mathrm{C}_{16} \mathrm{H}_{32} \mathrm{O}_{2}$ zukommen.

Von den bislang bekannten Säuren der genannten Formel unterscheidet sich die vorstehende Hexadecylsäure durch den Schmelzpunkt und die weiter unten zu beschreibenden Oxydationsprodukte, welche sie unter dem Einflusse von Kaliumpermanganat bildet. Demnach ist die vorstehende Hexadecylsäure mit den bis jetzt bekannten Säuren von den Formel $\mathrm{C}_{10} \mathrm{H}_{32} \mathrm{O}_{2}$, der Palmitinsäure und der Diheptylessigsäure $\mathrm{CH}\left(\mathrm{C}_{7} \mathrm{H}_{15}\right)_{2} \mathrm{CO}_{2} \mathrm{H}$ nicht identisch, sondern nur. isomer. Tavern $e^{1}$ ) erhielt bei der Reduction der Convolvulinolsäure eine Pentadecylsäure, $\mathrm{C}_{15} \mathrm{H}_{30} \mathrm{O}_{2}$ vom Schmelz. $48^{\circ}$. Die vorliegende Hexadecylsäure ist, wie es die weitere Oxydation der Oxysäure zeigen wird, mit der von Taverne isolirten Säure homolog.

\section{Oxydation der Jalapinolsäure mittelst Kalium- permanganat.}

$\mathrm{Zu}$ diesem $\mathrm{Zwecke}$ wurde Jalapinolsäure in alkalischer Lösung mit so viel Kaliumpermanganat versetzt, dass auf $1 \mathrm{Mol}$. der ersteren 1 Atom activer Sauerstoff in Reaction gelangte, um die bei dieser Zersetzung etwa auftretenden intermediären Produkte kennen zu lernen.

$20 \mathrm{Grm}$ Jalapinolsäure wurden mit ca. $200 \mathrm{Ccm}$. Wasser übergossen, $16 \mathrm{Grm}$. Kalihydrat hinzugegeben und zu dieser Mischung, welche vorher auf $50^{\circ}-60^{\circ}$ erwärmt war, allmählich eine Lösung von $8 \mathrm{Grm}$. Kaliumpermanganat in $200 \mathrm{Ccm}$. Wasser gebracht.

1) Dissertation S, 33, Heidelberg 1894 , 
Kromer: Beitrag z. Kenntniss der Jalapinolsäure. 457

Die Reaction vollzog sich verhältnissmässig schnell und war nach 3 Stunden beendet. Die Mischung wurde auf dem Dampfbade erhitzt und von den suspendirten Manganoxyden durch Filtration getrennt. Letztere wurden nochmals mit heissem Wasser anhaltend ausgewaschen und die erhaltenen Waschwässer mit dem Filtrat vereinigt.

Beim Sättigen der alkalischen Lösung mit Kohlensäure fiel eine kalihaltige Verbindung in weissen, flockigen Krystallen heraus, welche sich bei der näheren Untersuchung als ein saures Salz der unveränderten Jalapinolsäure erwies.

Durch Zersetzung dieser Verbindung mit Salzsäure wurde eine Säure erhalten, die den Schmelzp. $68^{\circ}-69^{\circ}$ hatte und bei $64^{\circ}-63^{\circ}$ erstarrte. (Jalapinolsäure schmolz bei $68^{\circ}$.)

$0,3232 \mathrm{Grm}$. der bei $110^{\circ}-120^{\circ}$ bis zum constanten Gewichte getrockneten Verbindung hinterliessen nach dem Verbrennen und Befeuchten des Rückstandes mit Schwefelsäure 0,0498 Grm. $\mathrm{K}_{2} \mathrm{SO}_{4}=6,91 \%$, K.

\section{$\mathbf{K}$}

Berechnet für $\mathrm{C}_{18} \mathrm{H}_{31} \mathrm{KO}_{3}+\mathrm{C}_{18} \mathrm{H}_{32} \mathrm{O}_{3}$ : Gefunden:

$$
6,70 \quad 6,91 \% \text {. }
$$

Die Gesammtmenge der Jalapinolsäure, die aus dem Kalium. salz abgeschieden wurde, betrug 18,1 Grm.

Das mit Kohlensäure gesättigte Filtrat, welches vom ausgeschiedenen Salze befreit worden war, wurde auf ein kleines Volumen eingedampft und, mit Schwefelsäure angesäuert, der Destillation mit Wasserdämpfen unterworfen.

Das Destillat war schwach sauer und lieferte nach dem Sättigen mit Pottasche, Eindampfen und Extrahiren des Rückstandes mit Alkohol und Verdunsten des letzteren 0,8 Grm. der Kaliumverbindung einer flüchtigen Säure.

Nach dem Geruch des Aethylesters, welcher durch Erwärmen des Salzes mit Aethylalkohol und Schwefelsäure erhalten wurde, konnte eine der Valeriansäuren, namentlich Methyläthylessigsäure vorausgesetzt werden.

Diese Säure entsteht, wie weiter gezeigt werden soll, bei energischer Oxydation aus der Jalapinolsäure in grösserer Quantität.

Die bei der Oxydation unverändert gebliebene Jalapinolsäure wurde nochmals mit einer solchen Quantität von Kaliumpermanganat der Reaction unterworfen, dass auf 1 Mol. der Säure 1 Atom Sauerstoff kam und mit dem Reactionsgemisch 
wie beschrieben verfahren. Das Resultat, welches hierbei erzielt wurde, deckte sich mit dem bei vorstehend angeführtem Versuche erhaltenen.

Da die angewandten Oxydationsbedingungen keine Sprengung der Kohlenstoffgruppirung im Molekül der Jalapinolsäure hervorriefen, wurde eine neue Menge von Jalapinolsäure mit so viel Permanganat behandelt, dass auf $1 \mathrm{Mol}$. der Säure 4 Atome activer Sauerstoff kamen.

$20 \mathrm{Grm}$. Jalapinolsäure wurden mit $32 \mathrm{Grm}$. Kalihydrat und ca. $200 \mathrm{Ccm}$. Wasser in Lösung gebracht, alsdann auf ca. $50^{\circ}$ erwärmt und eine Lösung von 32 Grm. Kaliumpermanganat in $400 \mathrm{Ccm}$. Wasser allmählich hinzugegeben.

Das Reactionsgemisch wurde durch Filtriren von den Oxyden des Mangans getrennt, und in das mit den Waschwässern vereinigte Filtrat Kohlensäure bis zur Sättigung geleitet. Auch hier schied sich das vorher erwähnte saure Kaliumsalz der Jalapinolsäure in weissen Flocken aus, aus welchen die Säure durch Salzsäure in Freiheit gesetzt wurde.

Die Säure erstarrte in den charakteristischen Krystallisationen der Jalapinolsäure, schmolz bei $67^{\circ}-68^{\circ}$ und erstarrte bei $63^{\circ}-62^{\circ}$. Aus Alkohol krystallisirt, wurde in kleiner Quantität eine Fraction erhalten, die bei $68^{\circ}-69^{\circ}$ schmolz und bei $64^{\circ}$ erstarrte, während die Hauptmenge aus dem bei $67^{\circ}-68^{\circ}$ schmelzenden Antheile bestand.

Wie weiter gezeigt werden soll, entstehen bei der Oxydation Säuren von höherem Schmelzpunkte, die ebenfalls in Wasser schwer löslich sind, und es ist sehr möglich, dass der den höheren Schmelzpunkt aufweisenden Fraction kleine Mengen dieser den Schmelzpunkt erhöhenden Säuren beigemischt waren.

Die Gesammtmenge der durch Kohlensäure abgeschiedenen Säure, welche der Oxydation entgangen war, betrug, im lufttrocknen Zustande gewogen, etwa $10 \mathrm{Grm}$.

Die mit Kohlensäure gesättigte, vom jalapinolsauren Salz abfiltrirte Flüssigkeit wurde zur Trockne verdampft und der Verdampfungsrückstand mit Alkohol anhaltend extrahirt.

Der in Alkohol lösliche Theil, welcher neben anderen Salzen die Kalisalze der flüchtigen Säuren enthalten musste, sowie der in genanntem Lösungsmittel unlösliche Theil, der 
Kromer: Beitrag z. Kenntniss der Jalapinolsäure. 459 neben Kaliumcarbonat noch Kaliverbindungen organischer Säuren, welche in Alkohol schwer oder unlöslich waren, enthalten konnte, wurden gesondert untersucht.

A) Der in Alkohol unlösliche Theil des Ver. da mpfung srü ckstan des wurde mit Schwefelsäure angesäuert und der Destillation mit überhitztem Wasserdampf unterworfen.

Das Destillat. reagirte neutral, aber der Destillationsrückstand schied beim Erkalten glänzende Blättchen aus, die, unter dem Mikroskop betrachtet, theils aus regelmässigen Rhomben, theils aus solchen, die in den der Diagonale entsprechenden Ecken abgestumpft waren, bestanden und somit, von der Oberfläche aus gesehen, sechsseitige Blättchen vorstellten.

Die Krystalle wurden abfiltrirt und die rückständige, schwefelsäurehaltige Flüssigkeit mit Aether ausgeschüttelt. Lotzteres Lösungsmittel hinterliess beim Verdunsten einen unbedeutenden Rückstand. Die Krystalle waren in Alkohol, Aether und kochendem Wasser schwer löslich. Beim Abkühlen einer heiss gesättigten Lösung schied sich die Substanz krystallinisch aus. Die alkoholische Lösung der Krystalle reagirte stark sauer und trieb aus den löslichen kohlensauren Salzen die Kohlensäure aus.

Zur Reinigung wurde die Säure mehrmals aus heissem Wasser umkrystallisirt. Die Gesammtmenge der erhaltenen Säure betrug etwa 1,8 Grm.

Die Säure schmolz im Capillarrohre bei $131^{\circ}-133^{\circ}$ und erstarrte bei $125,5-124^{\circ}$.

Das Silbersalz derselben wurde durch Fällung der Ammoniakverbindung mit Silbernitrat erhalten. Dasselbe war vollkommen weiss, lichtbeständig, im trocknen Zustande stark elektrisch und wurde zur Analyse über Schwefelsäure getrocknet.

$0,298 \mathrm{Grm}$. des Silbersalzes hinterliessen 0,1535 Grm. $\mathrm{Ag}=51,50 \% \mathrm{Ag}$. $0,201 \mathrm{Grm}$. derselben Verbindung gaben 0,1041 Grm. $\mathrm{Ag}=51,79 \% \mathrm{Ag}$.

\begin{tabular}{cccc} 
& Berechnet für & \multicolumn{2}{c}{ Gefunden: } \\
& $\mathrm{C}_{10} \mathrm{H}_{10} \mathrm{Ag}_{2} \mathrm{O}_{4}:$ & 1. & 2. \\
$\mathrm{Ag}$ & 51,92 & 51,51 & $51,79 \%$.
\end{tabular}

Die erhaltenen analytischen Werthe kommen einer Sebacinsäure von der Zusammensetzung $\mathrm{C}_{10} \mathrm{H}_{18} \mathrm{O}_{4}$ zu. Eine solche Säure vom Schmelzp. $127^{\circ}$ ist bei der Oxydation oder auch 
trocknen Destillation von vielen Fetten und fetten Oelen erhalten worden. Krafft und Nördlinger ${ }^{1}$, welche sie durch Destillation reinigten, fanden für dieselbe den Schmelzp. $133^{\circ}$ bis $133,5^{\circ}$.

Mayer ${ }^{2}$ ) erhielt die Sebacinsäure bei der Oxydation des Jalapenharzes mittelst Salpetersäure und nannte sie wegen des niederen Schmelzpunktes $\left(108^{\circ}-109^{\circ}\right)$ Ipomsäure. Taverne ${ }^{3}$ ) oxydirte Convolvulinolsäure mit Salpetersäure und erhielt eine Sebacinsäure vom Schmelzp. $116^{\circ}$. Hoehnel ${ }^{4}$ behandelte dieselbe Säure mit Salpetersäure sowie mit Kaliumpermanganat und gelangte in beiden Fällen zu einer Sebacinsäure, die übereinstimmend bei $108^{\circ}-109^{\circ}$ schmolz und die er Ipomsäure nannte.

B) Der in Alkohol lösliche Theïl des Verdampfungsrückstandes wurde vom Alkohol befreit und nach dem Ansäuern mit Schwefelsäure der Destillation mit Wasserdämpfen so lange unterworfen, bis das Destillat neutral reagirte.

Der im Kolben verbliebene Rückstand bestand aus gelben Oeltropfen, die beim Erkalten erstarrten, auch schied die Fliissigkeit einen weissen, flockigen Körper, der deutlich krystallinische Structur erkennen liess, in nicht unbedeutender Menge ab. Die Untersuchung des in Alkohol löslichen Theiles der Kaliumverbindungen, die bei der Oxydation der Jalapinolsäure erhalten waren, zerfiel somit in die Trennung der mit Wasserdämpfen flüchtigen Antheile und solcher Körper, die sich nicht bei der angegebenen Arbeitsmethode vertlüchtigten.

Das Destillat, welches sauer reagirte, wurde mit Kaliumcarbonat gesättigt, zur Trockne verdampft und der Rückstand mit Alkohol extrahirt. Der Rückstand von der Alkoholextraction war vollkommen weiss gefärbt und bestand aus den Kaliverbindungen der flüchtigen Säuren. Die Gesammtmenge derselben betrug etwa 4,0 Grm.

1) Ber. 22, 818 .

2) Ann. Chem. 83, 143; 95, 160.

3) Dissertation, S. 35. Heidelberg 1894.

4) Arch. d. Pharm. 234, Heft 9 (1896). 
Kromer: Beitrag z. Kenntniss der Jalapinolsäure. 461

Die aus dem Salze abgeschiedene Säure wurde in ihr Kalisalz übergeführt und alsdann mit Silbernitrat fractionirt gefällt.

I. Fraction:

0,3035 Grm. der getrockneten Verbindung hinterliessen 0,157 Grm. $\mathrm{Ag}=51,72 \% \mathrm{Ag}$.

II. Fraction:

0,2515 Grm. der Verbindung gaben $0,131 \mathrm{Grm} . \mathrm{Ag}=52,08 \%$ Ag.

III. Fraction:

$0,2618 \mathrm{Grm}$. derselben Verbindung gaben 0,140 Grm. $\mathrm{Ag}=53,47 \% \mathrm{Ag}$.

IV. Fraction:

$0,2240 \mathrm{Grm}$. derselben Verbindung gaben 0,1233 Grm. $\mathrm{Ag}=55,04 \% \mathrm{Ag}$.

V. Fraction:

$0,2647 \mathrm{Grm}$. derselben Verbindung gaben $0,1502 \mathrm{Grm}$. $\mathrm{Ag}=56,74 \% \mathrm{Ag}$.

Die Fractionen 1 und 2 waren vollkommen weiss und überwogen der Quantität nach die Fractionen 3, 4 und 5, letztere waren durch reducirtes Silber grau gefärbt. Das von der Fällung hinterbliebene Filtrat war durch reducirtes Silber getrübt.

Zur Darstellung eines Kalksalzes wurden die Silbersalze der Fällung 1 und 2 mit Schwefelsäure angesäuert und mit Aether anhaltend ausgeschüttelt. Dem Aether wurde die Säure durch kleine Mengen von Wasser entzogen, und letztere Flüssigkeit mit frischgefälltem Calciumcarbonat gesättigt. Die vom überschüssigen Calciumcarbonat abfiltrirte Flüssigkeit wurde auf ein kleines Volumen eingedampft und alsdann der Krystallisation überlassen.

Die Krystalle des Kalksalzes wurden durch Pressen zwischen Filtrirpapier von anhaftender Mutterlauge befreit und der Analyse unterzogen.

0,5155 Grm. der Calciumverbindung verforen im Exsiceator über Kalk und Schwefelsäure $0,0275 \mathrm{Grm} . \mathrm{H}_{2} \mathrm{O}$, hierauf bei $110^{\circ} 0,0356 \mathrm{Grm}$. $\mathrm{H}_{2} \mathrm{O}$, mithin im Ganzen $=0,0631$ Grm. $\mathrm{H}_{2} \mathrm{O}=12,24 \% \mathrm{H}_{2} \mathrm{O}$.

$0,4755 \mathrm{Grm}$. des bei $110^{\circ}$ getrockneten Kalksalzes gaben 0,2695 Grm. $\mathrm{CaSO}_{4}=16,66 \%$ Ca.

Den analytischen Werthen nach zu urtheilen, wie sie bei der Analyse des Silbersalzes der ersten Fällungen und des Kalksalzes erhalten worden sind, liegt hier eine der Valeriansäuren vor.

Berechnet für $\mathrm{C}_{6} \mathrm{H}_{9} \mathrm{AgO}_{2}$ : Für das Silberalz wurden erhalten: $\mathrm{Ag} \quad 51,67 \quad 51,72 \quad 52,08 \%$. 
462 Kromer: Beitrag z. Kenntniss der Jalapinolsänre.

$\begin{array}{ccc} & \text { Berechnet für }\left(\mathrm{C}_{5} \mathrm{H}_{9} \mathrm{O}_{2}\right)_{2} \mathrm{Ca}+2 \mathrm{H}_{2} \mathrm{O}: & \text { Das Kalksalz lieferte: } \\ \mathrm{H}_{2} \mathrm{O} & 12,94 & 12,24 \% \\ & \text { Berechnet für }\left(\mathrm{C}_{5} \mathrm{H}_{9} \mathrm{O}_{2}\right)_{2} \mathrm{Ca}: & \text { Das Kalksalz lieferte: } \\ \mathrm{Ca} & 16,53 & 16,66 \%\end{array}$

Zur weiteren Identificirung der erhaltenen Säure wurde die Löslichkeit des Silbersalzes in der Weise bestimmt, dass das sorgfältig ausgewaschene und abgepresste Salz, welches vollkommen weiss war, mit einer zur Lösung unvollständigen Menge Wasser auf dem Dampfbade längere Keit auf $100^{\circ}$ erhitzt wurde. Nachdem die Flüssigkeit heiss filtrirt worden war und bis zum Erkalten geschüttelt wurde, hatte sich aus derselben eine nicht unbedeutende Menge des Silbersalzes in weissen, nadelförmigen Krystallen abgeschieden. In dem Filtrate wurde alsdann das gelöste Silbersalz nach der Methode von Volhard durch Titration mittelst Rhodanlösung bestimmt.

Beim Erhitzen des gefällten als auch des aus wässriger Lösung krystallisirten Salzes mit Wasser trat stets eine Reduction des Salzes ein.

10 Cem. einer Lösung des durch Fällen aus dem Kalksalze bereiteten Silbersalzes verbrauchten $2,95 \mathrm{Ccm}$. einer Rhodanlösung, von der 1 Ccm. 0,011612 Grm. Ag oder 0,0224713 Grm. $\mathrm{C}_{5} \mathrm{H}_{0} \mathrm{AgO}_{2}$ făllte. Mithin enthielten $10 \mathrm{Ccm}$. obigex Lösung 0,066290335 Grm. Silbersalz oder 100 Theile Wasser lösten rund 0,663 Theile Salz.

$10 \mathrm{Ccm}$. einer Lösung des durch Krystallisation erbaltenen Salzes verbrauchten 2,4 Ccm. Rhodonlösung $=0,05393112 \mathrm{Grm}$. Salz; somit waren in 100 Theilen Lösung 0,539 Theile Salz.

Die von mir für dieses Salz gefundene Löslichkeit entspricht derjenigen, die H. Elte ow $^{1}$ ) für das methyläthylessigsaure Silber fand. Derselbe ermittelte, dass $100 \mathrm{Grm}$. einer wässrigen Lösung des genannten Salzes 0,575 Grm. Silbersalz enthalten. Conrad und Bischoff ${ }^{2}$ ) wiederum fanden für das genannte Silbersalz eine Löslichkeit von 1,128 Grm. in 100 Thln. Wasser.

Taverne ${ }^{3}$ ) erhielt bei der Oxydation der Convolvulinolsäure mittelst rauchender Salpetersäure eine optisch active Methyläthylessigsäure, von deren Silbersalz sich 1,13 Grm. in 100 Thln. Wasser lösten.

1) Journ. d. russ. chem. Gesellsch. 9, 163-177.

2) Ann. Chem. 204, 151.

3) Dissertation, Heidelberg 1894. 
Kromer: Beitrag ». Kenntniss der Jalipinolsäure. 463

Zum gleichen Resultate gelangte Hoehnel ${ }^{1}$ ), indem er für das von ihm isolirte Silbersalz eine Löslichkeit von 0,998 Grm. in $100 \mathrm{Ccm}$. Wasser fand.

Was die Eigenschaften des methyläthylessigsauren Calciums anbetrifft, so krystallisirt dasselbe nach den Untersuchungen

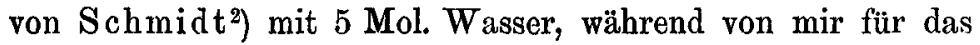
vorliegende Salz 2 Mol. Krystallwasser ermittelt worden sind.

D. Milojkovic ${ }^{3}$ beobachtete, dass das methyläthylessigsaure Calcium je nach der Temperatur Kalksalze mit 5,3 Mol. oder 1 Mol. Wasser zu bilden vermag.

Nach der Entfernung der Methyläthylessigsäure durch Destillation mit überhitzten Wasserdämpfen hinterblieben im Kolben, wie bereits oben erwähnt wurde, gelbe, ölige Tropfen, die beim Abkühlen erstarrten, wobei sich gleichzeitig aus der Flüssigkeit weisse, mikroskopische, zu Drusen vereinigte Krystalle abschieden. Die Flüssigkeit wurde vom ausgeschiedenen Antheile durch Filtration getrennt und das schwefelsäurehaltige Filtrat zur Extraction von etwa gelösten Körpern mit Aether ausgeschüttelt. Beim Verdunsten des Aethers hinterblieb eine kleine Menge eines weissen, fettartigen Körpers, die zur Hauptmenge hinzugegeben wurde.

Zur Trennung des Fettsäuregemenges wurde dasselbe auf dem Wasserbade von Wasser befreit und der Rückstand wiederholt mit kleinen Mengen Aether behandelt. Diese Operation wurde einigemal wiederholt, wobei ein in Aether schwer löslicher Rückstand, der sich als Sebacinsäure vom Schmelzpukte $132^{\circ}-132^{\circ}$ erwies, und ein in Aether leicht löslicher Antheil, der wiederum aus einem krystallinischen Antheil bestand, welcher von einer amorphen gelben Säure durchsetzt war, erhalten wurde.

Ein Versuch, die Isolirung der beiden Säuren auf Grund der Löslichkeit der Bleisalze in Aether oder Alkohol zu bewirken, führte zu keinem Resultate, da es sich zeigte, dass die Bleiverbindungen dieser Säuren hinsichtlich der Löslichkeit in den genannten Lösungsmitteln keine nennenswerthen Unterschiede zeigten.

1) Arch. d. Pharm. 234, 9. Heft 1896.

2) Ann. Chem. 208, 256. (1881.)

8) Nach Jahrbuch der organ. Chemie von Gaetano Minunni 1893. 
464 Kromer: Beitrag z. Kenntniss der Jalapinolsäure.

Wenngleich auch langwierig, so führte der Weg zum Ziele, dass das Säuregemenge wiederholt mit Wasser ausgekocht wurde. Die bei gewöhnlicher Temperatur schmierige Säure, in welcher keine Krystallisation erkannt werden konnte, löste sich nicht in Wasser und blieb in sehr geringer Menge zurück, so dass mit ihr keine Analyse ausgeführt werden konnte, während sich die krystallinische Säure beim Eindampfen und darauffolgendem Abkühlen der auf ein kleines Volumen eingedampften Lösung in weissen Krystalldrusen abschied.

Das Lösen und Abkühlen der Substanz in genannter Flüssigkeit wurde so lange wiederholt, bis keine Schwankungen in dem Schmelzpunkte mehr beobachtet werden konnten.

Die Säure war vollkommen weiss gefärbt, löste sich in Alkohol und Aether leicht und wurde zur Reinigung einigemal aus erstgenanntem Lösungsmittel umkrystallisirt.

Zur Analyse wurde sie auf dem Wasserbade geschmolzen, nach dem Erkalten fein zerrieben und im Exsiccator getrocknet. Im Ganzen wurden bei der Oxydation ca. 1,5 Grm. derselben abgeschieden. Die Säure schmolz bei $89^{\circ}-91^{\circ}$ und liess keinen scharf begrenzten Erstarrungspunkt erkennen.

Zur Gewinnung des Silbersalzes wurde die Säure mit Ammoniak neutralisirt und mit Silbersalpeter gefällt. Das Silbersalz stellte, mit kleinen Mengen Wasser ausgewaschen, alsdann im Exsiccator über Schwefelsäure getrocknet, ein weisses, lichtbeständiges, stark elektris ches Pulver vor.

0,334 Grm. des Silbersalzes gaben 0,1705 Grm. Ag $=51,04 \%$ Ag. Berechnet für $\mathrm{C}_{10} \mathrm{H}_{10} \mathrm{Ag}_{2} \mathrm{O}_{4}$ :

$$
\text { Ag } \quad 51,92 \quad 51,04 \% \text {. }
$$

1. $0,1961 \mathrm{Grm}$. der getrockneten Säure gaben $0,1563 \mathrm{Grm} . \mathrm{H}_{2} \mathrm{O}$ und 0,4177 Grm. $\mathrm{CO}_{2}=8,85 \% \mathrm{H}$ und $58,08 \% \mathrm{C}$.

2. 0,1665 Grm. derselben Substanz lieferten $0,1337 \mathrm{Grm} . \mathrm{H}_{2} \mathrm{O}$ und 0,356 Grm. $\mathrm{CO}_{2}=8,92 \% \mathrm{H}$ und $58,30 \% \mathrm{C}$.

\begin{tabular}{lccc} 
& Berechnet für & \multicolumn{2}{c}{ Gefunden: } \\
& $\mathrm{C}_{\mathbf{1 0}} \mathrm{H}_{18} \mathrm{O}_{\mathbf{4}}:$ & $\mathbf{1 .}$ & $\mathbf{2 .}$ \\
$\mathbf{3}$ & $\mathbf{5 9 , 4 0}$ & $\mathbf{5 8 , 0 8}$ & $\mathbf{5 8 , 3 0 \%}$ \\
$\mathrm{H}$ & 8,91 & 8,85 & $8,92 \%$
\end{tabular}

Wie die vorstehenden Zahlen beweisen, weicht der für diese Säure gefundene Kohlenstoffgehalt exheblich von dem 
Kromer: Beitrag z. Kenntniss der Jalapinolsäure. 465 $a b$, den eine Säure von der Zusammensetzung einer Sebacinsäure erfordert, während der Metallgehalt des Silbersalzes dieselbe wahrscheinlich macht.

Die Möglichkeit, dass hier eine nicht genügend gereinigte Säure oder ein Säuregemenge vorliegt, muss offen gelassen werden, weil die bei der Oxydation entstandene Quantität keine weiteren Versuche zuliess.

Es möge aber hier nochmals hervorgehoben werden, dass die aus heissem Wasser und aus Aether krystallisirte Säure weiss war und unter dem Mikroskope betrachtet in Drusen krystallisirte, also eine Krystallisation aufwies, die sich wesentlich von der isolirten Sebacinsäure unterschied.

Es ist höchst wahrscheinlich, dass die von Mayer ${ }^{1}$ ) bei der Oxydation des Jalapenharzes mittelst Salpetersäure', erhaltene Sebacinsäure von der Formel $\mathrm{O}_{10} \mathrm{H}_{18} \mathrm{O}_{4}$ und dem Schmelzpunkte $108^{\circ}-109^{\circ}$, die er Ipomsäure nannte, aus einem Gemenge der Sebacinsäure vom Schmelzpunkte 131 $\ldots 133^{\circ}$ und der vorstehenden Säure, die bei $89^{\circ}-91^{\circ}$ schmolz, bestand.

Nicht unerwähnt möge das gleichzeitige Auftreten von Methyläthylessigsäure und einer Sebacinsäure hinsichtlich des Umstandes sein, dass Pagenstecher ${ }^{2}$ ) eine derartige Säure vom Schmelzpunkte $184^{\circ}-194^{\circ}$ bei der Behandlung von Methylbromäthylessigsäure mittelst Natriumamalgam erhielt.

Diese Bildung, welche analog der Entstehung der Pinakone aus den Ketonen und Aldehyden ist, vollzieht sich nach der Ansicht Pagenstecher's bei augenblicklichem Mangel von Wasserstoff durch gegenseitige Sättigung von ungesättigten Resten.

Fasse ich die bei der vorstehenden Untersuchung erhaltenen Resultate zusammen, so ergiebt sich:

1. Die Jalapinolsäure ist eine Oxylexadecylsäure (Hexadekanolsäure) von der Zusammensetzung $\mathrm{O}_{16} \mathrm{H}_{32} \mathrm{O}_{3}$, die mit der normalen Hexadekanolsäure nicht identisch, sondern isomer ist.

2. Der Wasserstoff der Hydroxylgruppe kann durch Säurereste in der Weise ersetzt werden, dass auf die Ester der

1) A. a. 0 .

2) Ann. Chem. 195, 121.

Journal f. prakt. Chemie [2] Bd. 57. 
Oxysäure das Säureanhydrid einwirkt. Der erhaltene Ester von der Zusammensetzung $\left(\mathrm{C}_{15} \mathrm{H}_{30} . \mathrm{OCOCH}_{3} . \mathrm{CO}_{2} \mathrm{C}_{2} \mathrm{H}_{5}\right)$ war flüssig und destillirte bei vermindertem Druck ohne Zersetzung.

3. Die Oxysäure addirt kein Brom, doch ist sie im Stande, Brom unter Substitution aufzunehmen und ein flüssiges Bromprodukt zu liefern, das sich nicht einheitlicher Natur erwies.

4. Durch Reduction mit Jodwasserstoff wird die Oxysäure in eine Hexadecylsäure $\mathrm{C}_{10} \mathrm{H}_{32} \mathrm{O}_{2}$ übergeführt, die mit den bislang bekannten Säuren dieser Formel nicht identisch, sondern nur isomer ist.

Durch Oxydation mittelst Kaliumpermanganat in alkalischer Lösung wird die Jalapinolsäure in Methyläthylessigsäure, Sebacinsäure und in eine mit der Sebacinsäure vielleicht isomere Säure vom Schmelzpunkt $89^{\circ}-91^{\circ}$ zerlegt.

Den erhaltenen Resultaten nach kann die Constitution der Jalapinolsäure = Oxyhexadecylsäure durch folgende Formel veranschaulicht werden:

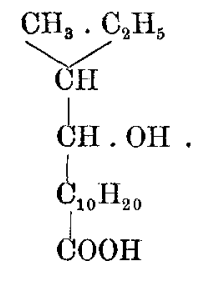

Es ist mir eine angenehme Pflicht, Herrn Prof. Alex. Saytzeff an dieser Stelle für das mir zu Theil gewordene liebenswürdige Entgegenkommen und die seltene Bereitwilligkeit, mit welcher er mir sein Laboratorium zur Verfügung stellte, meinen Dank auszusprechen. 